Article

\title{
Ritz Method in Vibration Analysis for Embedded Single-Layered Graphene Sheets Subjected to In-Plane Magnetic Field
}

\author{
Olga Mazur ${ }^{1,2, *(\mathbb{D})}$ and Jan Awrejcewicz ${ }^{1}$ \\ 1 Biomechanics and Mechatronics, Department of Automation, Lodz University of Technology, 90-924 Lodz, \\ Poland; jan.awrejcewicz@p.lodz.pl \\ 2 Department of Applied Mathematics, National Technical University Kharkiv Polytechnic Institute, \\ Kyrpychova Str 2, 61002 Kharkiv, Ukraine \\ * Correspondence: olga.mazur@p.lodz.pl
}

Received: 19 February 2020; Accepted: 27 March 2020; Published: 2 April 2020

\begin{abstract}
Vibrations of single-layered graphene sheets subjected to a longitudinal magnetic field are considered. The Winkler-type and Pasternak-type foundation models are employed to reproduce the surrounding elastic medium. The governing equation is based on the modified couple stress theory and Kirchhoff-Love hypotheses. The effect of the magnetic field is taken into account due to the Lorentz force deriving from Maxwell's equations. The developed approach is based on applying the Ritz method. The proposed method is tested by a comparison with results from the existing literature. The numerical calculations are performed for different boundary conditions, including the mixed ones. The influence of the material length scale parameter, the elastic foundation parameters, the magnetic parameter and the boundary conditions on vibration frequencies is studied. It is observed that an increase of the magnetic parameter, as well as the elastic foundation parameters, brings results closer to the classical plate theory results. Furthermore, the current study can be applied to the design of microplates and nanoplates and their optimal usage.
\end{abstract}

Keywords: Ritz method; modified couple stress theory; magnetic field; elastic foundation

\section{Introduction}

The application of micro and nanoscale structures in the high tech industry as elements of nano-electromechanical systems (NEMS), micro-electromechanical systems (MEMS), resonators, sensors, energy storage systems, DNA detectors, drug delivery [1-5] has been attracted the interest of scientists. The above-mentioned applications of micro/nanoplates are associated with their excellent mechanical, electrical, magnetic and chemical properties. It results from the study of the vibrational characteristics of micro/nanoplates that the ways of their optimization play an important role. The experimental and theoretical investigations lead to a conclusion that when the thickness of the element is in a micro or nanoscale, a size dependence effect of a material appears and significantly affects the mechanical behavior of such objects [6-8] and the classical theory for a small-scale structure analysis can not be used. Thus, in the study of the micro and nano-elements, higher-order continuum theories have been applied, for example, the theory of micropolar elasticity by Cosserat and Cosserat [9], the couple stress theory by Mindlin and Tiersten [10], Toupin [11], Koiter [12], the nonlocal elasticity theory by Eringen [13], strain gradient theory by Lam et al. [6] and the modified couple stress theory proposed by Yang et al. [14].

Recently, the modified couple stress theory was employed to various small-scale plate and beam problems. For instance, Tsiatas in [15] proposed a new Kirchhoff plate model for the static analysis 
of isotropic microplates. Yin et al. [16], Simsek et al. [17], Jomehzadeh et al. [18] employed the modified couple stress theory to vibrations analysis of rectangular and circular microplates with various boundary conditions. Moreover, Tsiatas and Yiotis [19] applied the modified couple stress theory to an orthotropic plate analysis. Ziaee [20] explored the linear vibrations of a rectangular plate with an internal square hole in a thermal environment using the Ritz method. Investigation of bending, buckling and vibrations of an orthotropic skew plate was performed by Tsiatas and Yiotis [21].

Furthermore, the investigation regarding the embedded plate behavior includes the work of Akgöz and Civalek [22] (where the free vibrations of a single-layered graphene sheet resting on an elastic matrix as the Pasternak foundation are investigated), the work of Bastami [23] (where the nonlocal elasticity theory is used and the proposed approach is based on the Ritz method). Mohammadi [24,25] investigated thermo-mechanical vibrations and vibrations under the biaxial in-plane preload of orthotropic graphene sheet embedded in an elastic medium based on the nonlocal elasticity theory. Behfar and Naghdabadi in [26] studied vibrations of multi-layered nanoplates embedded into the elastic medium with the constant Van der Waals force acting between nanoplates.

The action of the magnetic field has a significant influence on the exposed micro and nanostructure. This fact was shown experimentally for carbon nanotubes by Choi et al. [27], Lee et al. [28] and graphenes by Faugeras et al. [29], Wang et al. [30]. The numerical investigation regarding the effect of the magnetic force on vibration characteristics of the micro/nanoplate is performed in a large number of studies. Transverse vibrations of embedded single-layered graphene sheets affected by an in-plane magnetic field were studied by Murmu et al. [31], Kiani [32], using the nonlocal elasticity theory. Ghorbanpour Arani et al. [33] investigated the 2D-magnetic field and biaxial in-plane preload effects on the vibration of double-bonded orthotropic graphene sheets, whereas orthotropic double-nanoplate system subjected to an in-plane magnetic field was analyzed by Atanasov et al. [34].

Analysis of published results has shown that the vibrations of an embedded graphene sheet subjected to a magnetic influence were studied within the nonlocal elasticity theory. However, the literature review indicates the novel results of the investigation associated with the modified couple stress theory. The study requires an account of a two-parameter elastic foundation, which is a combination with the acting Lorentz force can have an effect on the studied characteristics. In this paper we will apply the Ritz method for small-scale linear vibrations of plates. It should be noted that the application of the Ritz method allows us to study micro and nano rectangular plates in a magnetic field satisfying various boundary conditions, including the mixed ones (for example, two sides are simply supported and two sides are clamped), while reviewed works [31-34] are restricted to the study of the plates with the simply supported boundary conditions. It is important to note that in our work we presented the variational formulation of the considered problem.

The mathematical formulation of the problem is based on the modified couple stress theory and the Kirchhoff-Love hypotheses. In order to simulate the surrounding elastic medium, the Winkler-type and the Pasternak-type foundation models are used.

The paper is organized in the following way. In the first section, a short review of the existing results is outlined. The mathematical statement of the problem is given in the second section, whereas the next section is aimed at describing an action of the Lorentz force. The application of the Ritz method is reported in the fourth section and linear frequencies of small-scale plates obtained by the Navier method are presented in the fifth section. Results of a verification and numerical analysis are provided in the sixth section. The last section is aimed at the description of conclusions.

\section{Formulation of the Problem}

The present study is based on the modified couple stress theory which contains only one additional material length scale parameter and a symmetric couple stress tensor. According to this theory, the strain energy of an orthotropic plate has the following form

$$
\mathbf{U}=\frac{1}{2} \int_{V}\left(\sigma_{i j} \varepsilon_{i j}+m_{i j} \chi_{i j}\right) d V, i, j=x, y, z,
$$


where $\sigma_{i j}, \varepsilon_{i j}, m_{i j}, \chi_{i j}$ are components of stress tensor, strain tensor, diviatory part of the couple stress tensor and symmetric curvature tensor, respectively. Moreover, in (1) components of strain tensor are defined as

$$
\varepsilon_{i j}=\frac{1}{2}\left(u_{i, j}+u_{j, i}\right),
$$

where vector $U=\left(u_{x}, u_{y}, u_{z}\right)$ stands for the vector of displacements. The components of curvature tensor are

$$
\chi_{i j}=\frac{1}{2}\left(\theta_{i, j}+\theta_{j, i}\right)
$$

with

$$
\theta_{i}=\frac{1}{2} e_{i j k} u_{k, j}
$$

and $e_{i j k}$ stands for a permutation symbol. Based on the Kirchhoff-Love theory, displacements of the plate in $x, y$ and $z$ directions have the following form

$$
\begin{gathered}
u_{x}(x, y, z, t)=-z \frac{\partial w(x, y, t)}{\partial x}, u_{y}(x, y, z, t)=-z \frac{\partial w(x, y, t)}{\partial y}, \\
u_{z}(x, y, z, t)=w(x, y, t),
\end{gathered}
$$

where $w(x, y, t)$ is displacement of points of the middle plane in $z$ direction and $t$ denotes time. The strain-displacement relations are expressed as

$$
\varepsilon_{x x}=-z \frac{\partial^{2} w}{\partial x^{2}}, \varepsilon_{y y}=-z \frac{\partial^{2} w}{\partial y^{2}}, \varepsilon_{x y}=-2 z \frac{\partial^{2} w}{\partial x \partial y} .
$$

The components of the rotation vector (4) taking into account (5) are as follows

$$
\theta_{x}=\frac{\partial w}{\partial y}, \theta_{y}=-\frac{\partial w}{\partial x}, \theta_{z}=0
$$

and components of the curvature tensor (3) take the form

$$
\chi_{x x}=\frac{\partial^{2} w}{\partial x \partial y}, \chi_{y y}=-\frac{\partial^{2} w}{\partial x \partial y}, \chi_{x y}=\frac{1}{2}\left(\frac{\partial^{2} w}{\partial y^{2}}-\frac{\partial^{2} w}{\partial x^{2}}\right), \chi_{x z}=\chi_{y z}=\chi_{z z}=0 .
$$

The constitutive relations for an orthotropic plate have the following form

$$
\begin{gathered}
\sigma_{x x}=\frac{E_{1}}{1-v_{1} v_{2}}\left(\varepsilon_{x x}+v_{2} \varepsilon_{y y}\right), \sigma_{y y}=\frac{E_{2}}{1-v_{1} v_{2}}\left(\varepsilon_{y y}+v_{1} \varepsilon_{x x}\right), \sigma_{x y}=G \varepsilon_{x y}, \\
m_{i j}=2 G l^{2} \chi_{i j} .
\end{gathered}
$$

In the last expressions $E_{1}, E_{2}$ are Young's modules, $v_{1}, v_{2}$ are Poisson's ratios with $v_{1} E_{2}=v_{2} E_{1}$, $G$ is shear modulus, $l$ stands for the material length scale parameter. Thus, the strain energy of an orthotropic microplate can be expressed with respect to displacements in the following way

$$
\begin{aligned}
\mathbf{U}=\frac{1}{2} \iint_{\Omega}\left(\left(D_{11}+D_{L}\right)\right. & \left(\frac{\partial^{2} w}{\partial x^{2}}\right)^{2}+\left(D_{22}+D_{L}\right)\left(\frac{\partial^{2} w}{\partial y^{2}}\right)^{2}+2\left(D_{12}-D_{L}\right) \frac{\partial^{2} w}{\partial x^{2}} \frac{\partial^{2} w}{\partial y^{2}}+ \\
& \left.+4\left(D_{66}+D_{L}\right)\left(\frac{\partial^{2} w}{\partial x \partial y}\right)^{2}\right) d x d y
\end{aligned}
$$

where

$$
D_{11}=\frac{E_{1} h^{3}}{12\left(1-v_{1} v_{2}\right)}, D_{22}=\frac{E_{2} h^{3}}{12\left(1-v_{1} v_{2}\right)}, D_{12}=v_{1} D_{22}, D_{66}=\frac{G h^{3}}{12}, D_{L}=l^{2} G h .
$$


In (11) $h$ denotes the thickness of the plate. Using Hamilton's principle, the governing equation describing vibration process of micro-scale plates resting on the two parameters foundation and subjected to transverse external load, can be obtained in the following form:

$$
\begin{gathered}
\left(D_{11}+D_{L}\right) \frac{\partial^{4} w}{\partial x^{4}}+\left(D_{22}+D_{L}\right) \frac{\partial^{4} w}{\partial y^{4}}+2\left(D_{12}+2 D_{66}+D_{L}\right) \frac{\partial^{4} w}{\partial x^{2} y^{2}}+ \\
+k_{W} w-k_{G_{x}} \frac{\partial^{2} w}{\partial x^{2}}-k_{G_{y}} \frac{\partial^{2} w}{\partial y^{2}}=q_{l}-\rho h \frac{\partial^{2} w}{\partial t^{2}}
\end{gathered}
$$

where $k_{W}$ and $k_{G_{x}}, k_{G_{y}}$ are the Winkler and shear modules of the elastic surrounding medium, respectively, $q_{l}$ is the external load, $\rho$ is the density of the plate.

Equation (12) is supplemented with the boundary conditions. The part of the boundary is supposed to be simply supported:

$$
w=0, M_{n}=0
$$

and clamped:

$$
w=0, \frac{\partial w}{\partial n}=0
$$

In (13) and (14) $M_{n}$ is a bending moment, whereas $n$ stands for a normal vector to the boundary.

\section{Influence of the Magnetic Field}

The choice of an appropriate external load plays a crucial role in changing the vibration characteristics of the considered micro/nanoplate. The necessary effect can be achieved by exposing the plate to a magnetic field. In this paper the plate subjected to the in-plane uni-axial magnetic field [31-34], see Figure 1, defined by the vector of magnetic field strength

$$
H=\left(H_{x}, 0,0\right)
$$

is considered. Distributing vector of the magnetic field $\vec{h}$ is defined using Maxwell's relations:

$$
\vec{h}=[\nabla,[\vec{U}, \vec{H}]] .
$$

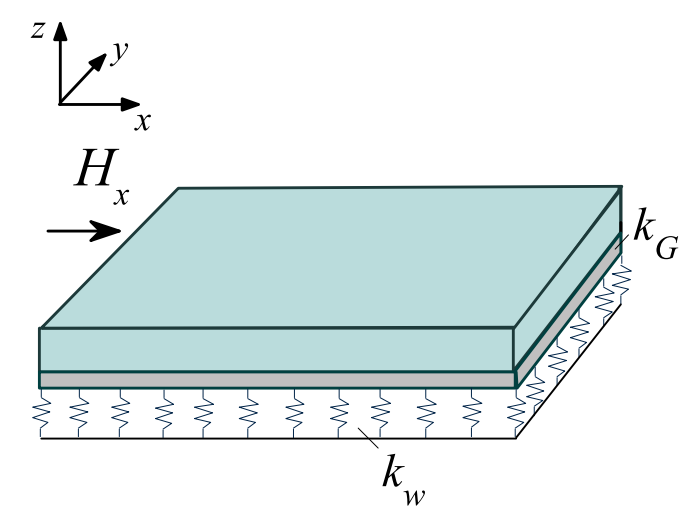

Figure 1. Rectangular plate resting on an elastic foundation.

Substituting (15) into (16) gives the following form of the distributing vector:

$$
\vec{h}=\left(-H_{x} \frac{\partial u_{y}}{\partial y}-H_{x} \frac{\partial u_{z}}{\partial z}, H_{x} \frac{\partial u_{y}}{\partial x}, H_{x} \frac{\partial u_{z}}{\partial z}\right) .
$$


The current density $\vec{J}$ is defined as follows

$$
\vec{J}=[\nabla, \vec{h}]
$$

and can be expressed as

$$
\begin{aligned}
\vec{J}= & \left(H_{x} \frac{\partial^{2} u_{z}}{\partial x \partial y}-H_{x} \frac{\partial^{2} u_{y}}{\partial x \partial z},-H_{x} \frac{\partial^{2} u_{z}}{\partial x^{2}}-H_{x} \frac{\partial^{2} u_{y}}{\partial y \partial z}-\right. \\
& \left.-H_{x} \frac{\partial^{2} u_{z}}{\partial z^{2}}, H_{x} \frac{\partial^{2} u_{y}}{\partial x^{2}}+H_{x} \frac{\partial^{2} u_{y}}{\partial y^{2}}+H_{x} \frac{\partial^{2} u_{z}}{\partial y \partial z}\right) .
\end{aligned}
$$

Thus, the Lorentz force can be written as

$$
f=\left(f_{x}, f_{y}, f_{z}\right)=\eta[\vec{J}, \vec{H}],
$$

where $\eta$ is the magnetic permeability. It should be pointed out that the transverse vibrations are studied, so we have taken into account the third coordinate $f_{z}$. Formula (20) yields

$$
f_{z}=\eta H_{x}^{2}\left(\frac{\partial^{2} u_{z}}{\partial x^{2}}+\frac{\partial^{2} u_{y}}{\partial y \partial z}+\frac{\partial^{2} u_{z}}{\partial z^{2}}\right) .
$$

Reflecting Kirchhoff-Love hypotheses, the transverse component $f_{z}$ is recast to the following form

$$
f_{z}=\eta H_{x}^{2}\left(\frac{\partial^{2} w}{\partial x^{2}}-\frac{\partial^{2} w}{\partial y^{2}}\right)
$$

Thus, the magnetic field produces the force expressed by the following formula

$$
q_{l}=\int_{\frac{-h}{2}}^{\frac{h}{2}} f_{z} d z=\eta H_{x}^{2} h\left(\frac{\partial^{2} w}{\partial x^{2}}-\frac{\partial^{2} w}{\partial y^{2}}\right) .
$$

\section{Application of the Ritz Method}

The variational formulation of the considered problem using (10), (23) and carrying out the integration by parts yields the following functional:

$$
\begin{gathered}
\int_{\frac{-a}{2}}^{\frac{a}{2}} \int_{\frac{-b}{2}}^{\frac{b}{2}}\left[\left(D_{11}+D_{L}\right)\left(\frac{\partial^{2} w}{\partial x^{2}}\right)^{2}+\left(D_{22}+D_{L}\right)\left(\frac{\partial^{2} w}{\partial y^{2}}\right)^{2}+2\left(D_{12}-D_{L}\right) \frac{\partial^{2} w}{\partial x^{2}} \frac{\partial^{2} w}{\partial y^{2}}+\right. \\
+4\left(D_{66}+D_{L}\right)\left(\frac{\partial^{2} w}{\partial x \partial y}\right)^{2}+\eta H_{x}^{2} h\left(\left(\frac{\partial w}{\partial x}\right)^{2}-\left(\frac{\partial w}{\partial y}\right)^{2}\right)+ \\
\left.+k_{W} w^{2}+k_{G_{x}}\left(\frac{\partial w}{\partial x}\right)^{2}+k_{G_{y}}\left(\frac{\partial w}{\partial y}\right)^{2}-\rho h w^{2}\right] d x d y .
\end{gathered}
$$

According to the Ritz method, the deflection of the plate is represented as

$$
w=\sum_{i=1}^{n} c_{i} w_{i}(x, y)
$$


In (25) $c_{i}$ stand for unknown coefficients, $w_{i}=g \phi_{i}$, and $g$ is shape function selected depending on the boundary conditions and shape of the plate, whereas $\phi_{i}$ is a system of the power polynomials. For the rectangular plate with sides $a$ and $b$, the shape function is taken as

$$
g(x, y)=\left(x+\frac{a}{2}\right)^{p}\left(x-\frac{a}{2}\right)^{q}\left(y+\frac{b}{2}\right)^{r}\left(y-\frac{b}{2}\right)^{s},
$$

where $p, q, r, s$ depend on the boundary conditions. In order to find minimum of the functional (24), we substituted (25) in (24), and the partial derivatives with respect to unknown coefficients are equated to zero, giving the following algebraic system of equations

$$
\left(\left\{k_{i j}\right\}-\omega^{2}\left\{m_{i j}\right\}\right)\left\{c_{i}\right\}=0,
$$

where elements of the matrices are defined as

$$
\begin{gathered}
k_{i j}=\int_{-a / 2}^{a / 2} \int_{-b / 2}^{b / 2}\left[\left(D_{11}+D_{L}\right) \frac{\partial^{2} w_{i}}{\partial x^{2}} \frac{\partial^{2} w_{j}}{\partial x^{2}}+\left(D_{22}+D_{L}\right) \frac{\partial^{2} w_{i}}{\partial y^{2}} \frac{\partial^{2} w_{j}}{\partial y^{2}}+\right. \\
+\left(D_{12}-D_{L}\right)\left(\frac{\partial^{2} w_{i}}{\partial x^{2}} \frac{\partial^{2} w_{j}}{\partial y^{2}}+\frac{\partial^{2} w_{i}}{\partial y^{2}} \frac{\partial^{2} w_{j}}{\partial x^{2}}\right)+4\left(D_{66}+D_{L}\right) \frac{\partial^{2} w_{i}}{\partial x \partial y} \frac{\partial^{2} w_{j}}{\partial x \partial y}+ \\
\left.+k_{W} w_{i} w_{j}+k_{G_{x}} \frac{\partial w_{i}}{\partial x} \frac{\partial w_{j}}{\partial x}+k_{G_{y}} \frac{\partial w_{i}}{\partial y} \frac{\partial w_{j}}{\partial y}+\eta H_{x}^{2} h\left(\frac{\partial w_{i}}{\partial x} \frac{\partial w_{j}}{\partial x}-\frac{\partial w_{i}}{\partial y} \frac{\partial w_{j}}{\partial y}\right)\right] d x d y, \\
m_{i j}=\rho h \int_{-a / 2}^{a / 2} \int_{-b / 2}^{b / 2} w_{i} w_{j} d x d y, i, j=1 . . n .
\end{gathered}
$$

System (27) has nonzero solutions if its determinant is 0 , which gives an equation for determining the frequency spectrum.

\section{Solution by the Navier Method}

According to the Navier method, the deflection of simply supported rectangular graphene sheet with length of the sides $a$ and $b$ is taken as in [24,35], and the linear frequency of the embedded small-scale plate effecting by in-plane uni-axial magnetic field can be calculated by the following formula

$$
\begin{aligned}
& \omega_{m n}^{2}== \frac{1}{\rho h}\left[\left(D_{11}+D_{L}\right)\left(\frac{m \pi}{a}\right)^{4}+\left(D_{22}+D_{L}\right)\left(\frac{n \pi}{b}\right)^{4}+\right. \\
&+2\left(D_{12}+2 D_{66}+D_{L}\right)\left(\frac{m \pi}{a}\right)^{2}\left(\frac{n \pi}{b}\right)^{2}+ \\
&\left.+k_{W}+k_{G_{x}}\left(\frac{m \pi}{a}\right)^{2}+k_{G_{y}}\left(\frac{n \pi}{b}\right)^{2}+\eta h H_{x}^{2}\left(\left(\frac{m \pi}{a}\right)^{2}-\left(\frac{n \pi}{b}\right)^{2}\right)\right] .
\end{aligned}
$$

\section{Results and Discussions}

In this work we investigated the model studied in [31,32], but in a contrast to the mentioned works, we used the modified couple stress theory, which allowed us to obtain the new results. In order to establish the validity of the current work we considered several vibration problems, which represent a simplification of the current model by using the classical plate theory (the material length scale parameter is neglected) and vanishing the magnetic influence. For each considered problem analysis of convergence of the results was performed and it was made a conclusion about a sufficient amount of terms in the series expansion (25). In Table 1 frequencies of the isotropic square plate with various boundary conditions are presented. Calculations are performed for the following types of the boundary conditions: all edges are simply supported (SSSS), three edges are simply supported and one is clamped (SSSC), two opposite sides are simply supported, the other two ones are clamped (SCSC), two opposite sides are simply supported, one is clamped and one is free (SCSF), the sides except one are simply 
supported and one is free (SSSF). We assumed that $k_{G_{x}}=k_{G_{y}}=k_{G}$ [25]. In this study, the dimensionless frequency parameter and the dimensionless Winkler and shear parameters are defined as follows

$$
\Omega=\omega a^{2} \sqrt{\frac{\rho h}{D_{11}}}, K W=k_{W} \frac{a^{4}}{D_{11}}, K G=k_{G} \frac{a^{2}}{D_{11}} .
$$

Analysis was performed for $v=0.3, h / a=0.01, l / h=0$. Here it should be noted that for isotropic case $E=E_{1}=E_{2}, v=v_{1}=v_{2}, G=E /(2(1+v))$. The solution by the Ritz method proposed in the paper is denoted as RS.

Table 1. Dimensionless frequencies $\Omega$ of isotropic square plate for various foundation parameters and boundary conditions.

\begin{tabular}{cccccccc}
\hline Method & KW & KG & SSSS & SSSC & SCSC & SCSF & SSSF \\
\hline [36] & & & 19.737 & 23.643 & 28.944 & 12.693 & 11.69 \\
[37] & & 0 & 19.735 & 23.659 & 28.995 & - & 11.677 \\
RS & & & 19.739 & 23.646 & 28.949 & 12.687 & 11.418 \\
[36] & & & 48.615 & 51.318 & 54.674 & 37.977 & 37.152 \\
[37] & 0 & \multirow{2}{*}{100} & 48.547 & 51.253 & 54.617 & - & 37.102 \\
RS & & & 48.615 & 51.323 & 54.679 & 37.981 & 37.129 \\
[36] & & & 141.873 & 144.2 & 146.719 & 112.481 & 111.745 \\
[37] & & \multirow{2}{*}{1000} & 140.182 & 142.439 & 144.877 & - & 110.424 \\
RS & & & 141.873 & 144.479 & 146.74 & 112.672 & 111.746 \\
[36] & & & 22.126 & 25.671 & 30.623 & 16.149 & 15.383 \\
[37] & & 0 & 22.125 & 25.687 & 30.672 & - & 15.373 \\
RS & & & 22.127 & 25.673 & 30.628 & 16.155 & 15.178 \\
[36] & & & 49.633 & 52.283 & 55.581 & 39.272 & 38.474 \\
[37] & \multirow{2}{*}{100} & \multirow{2}{*}{100} & 49.566 & 52.22 & 55.524 & - & 38.426 \\
RS & & & 49.633 & 52.289 & 55.586 & 39.276 & 38.453 \\
[36] & & & 142.225 & 144.547 & 147.06 & 112.925 & 112.192 \\
[37] & & 1000 & 140.538 & 142.789 & 145.222 & - & 110.876 \\
RS & & & 142.225 & 144.824 & 147.081 & 113.115 & 112.193 \\
[36] & & & 37.276 & 39.483 & 42.869 & 34.075 & 33.714 \\
[37] & & 0 & 37.274 & 39.493 & 42.902 & - & 33.708 \\
RS & & & 37.277 & 39.485 & 42.873 & 34.073 & 33.621 \\
[36] & & & 57.995 & 60.278 & 63.160 & 49.419 & 48.789 \\
[37] & 1000 & \multirow{2}{*}{100} & 57.936 & 60.222 & 63.109 & - & 48.749 \\
RS & & & 57.995 & 60.283 & 63.165 & 49.420 & 48.771 \\
[36] & & & 145.355 & 147.627 & 150.088 & 116.842 & 116.134 \\
[37] & & \multirow{2}{*}{1000} & 143.704 & 145.906 & 148.288 & - & 114.862 \\
RS & & & 145.355 & 147.899 & 150.109 & 117.026 & 116.135 \\
\hline
\end{tabular}

The orthotropic square plate with a free edge and others clamped edges are studied for the following mechanical and geometrical data [21]:

$$
D_{22} / D_{11}=1 / 2, D_{12} / D_{11}=0.3, D_{66} / D_{11}=1 / 3, v_{2}=0.3, h=100 \mu m, h / a=0.01 .
$$

The obtained results are reported in Table 2. 
Table 2. Dimensionless frequencies $\Omega$ of the orthotropic square small-scale plate for different thickness ratios.

\begin{tabular}{ccccc}
\hline Method & $\boldsymbol{l} / \boldsymbol{h}$ & $\omega_{\mathbf{1}}$ & $\omega_{\mathbf{2}}$ & $\omega_{\mathbf{3}}$ \\
\hline$[21]$ & & 17.543 & 36.034 & 45.660 \\
[38] & 0 & 17.860 & 36.295 & 45.683 \\
RS & 0 & 17.880 & 36.299 & 45.704 \\
[21] & 0.1 & 18.432 & 37.326 & 47.920 \\
RS & & 18.687 & 37.441 & 47.734 \\
[21] & 0.2 & 20.697 & 40.921 & 53.832 \\
RS & & 20.802 & 40.629 & 53.060 \\
[21] & 0.3 & 23.745 & 46.228 & 62.111 \\
RS & & 23.717 & 45.350 & 60.470 \\
[21] & \multirow{2}{*}{0.4} & 27.258 & 52.708 & 71.983 \\
RS & & 27.114 & 51.122 & 69.194 \\
\hline
\end{tabular}

In the next case study we considered the simply supported orthotropic rectangular graphene sheet with the following mechanical parameters [24]:

$$
E_{1}=1765 \mathrm{GPa}, E_{2}=1588 \mathrm{GPa}, \mathrm{G}=678.85 \mathrm{GPa}, \rho=2300 \mathrm{~kg} / \mathrm{m}^{3}, v_{1}=0.3, v_{2}=0.27,
$$

and the geometrical parameters $h=100 \mu m, h / a=0.01, b / a=1.5$. Table 3 contains the values of dimensionless frequency $\Omega$ obtained for the magnetic parameter $M P=0,25,50$, where the dimensionless magnetic parameter is introduced as follows

$$
M P=\frac{\eta h H_{x}^{2} a^{2}}{D_{11}}
$$

and the dimensionless Winkler and shear modules $K W=100, K G=10$. The results are calculated by the Ritz (RS) and Navier (NS) methods.

Table 3. Dimensionless frequencies $\Omega$ of the orthotropic rectangular small-scale plate for different thickness ratios and magnetic parameters, SSSS.

\begin{tabular}{lccccccc}
\hline Method & MP & \multicolumn{7}{c}{$l / h$} \\
\hline & & 0 & 0.2 & 0.4 & 0.6 & 0.8 & 1 \\
RS & \multirow{2}{*}{0} & 21.0203 & 21.8251 & 24.0784 & 27.4257 & 31.5203 & 36.1088 \\
NS & & 21.0205 & 21.8253 & 24.0788 & 27.4262 & 31.5209 & 36.1096 \\
RS & \multirow{2}{*}{25} & 24.0608 & 24.767 & 26.7739 & 29.8201 & 33.6244 & 37.9594 \\
NS & & 24.0612 & 24.7674 & 26.7744 & 29.8207 & 33.6251 & 37.9602 \\
RS & \multirow{2}{*}{50} & 26.7581 & 27.3948 & 29.2218 & 32.0361 & 35.6043 & 39.7239 \\
NS & & 26.7585 & 27.3953 & 29.2223 & 32.036 & 35.6051 & 39.7248 \\
\hline
\end{tabular}

For a rectangular plate with mechanical properties (32) and $h=100 \mu m, h / a=0.01, b / a=1.5$, the effect of magnetic field was investigated versus the thickness ratio $l / h$. The results are presented for plates with all simply supported edges (Figure 2) and clamped edges (Figure 3). In both cases, it can be seen an increase in the dimensionless frequency $\Omega(30)$, with an increase of both: the magnetic parameter as well as the material length scale parameter. The similar influence of the magnetic field was reported in [31,32] based on the nonlocal elasticity theory for simply supported boundary conditions. The largest values of frequency parameter are characteristic of the plate with clamped edges. Herewith an increase within the magnetic parameter leads to a decrease in the difference in the results between the classical and the modified couple stress theory. Thus, inter-atomic bonds of the micro/nanoplates influence the vibration behaviour less for higher values of the magnetic field strength. 


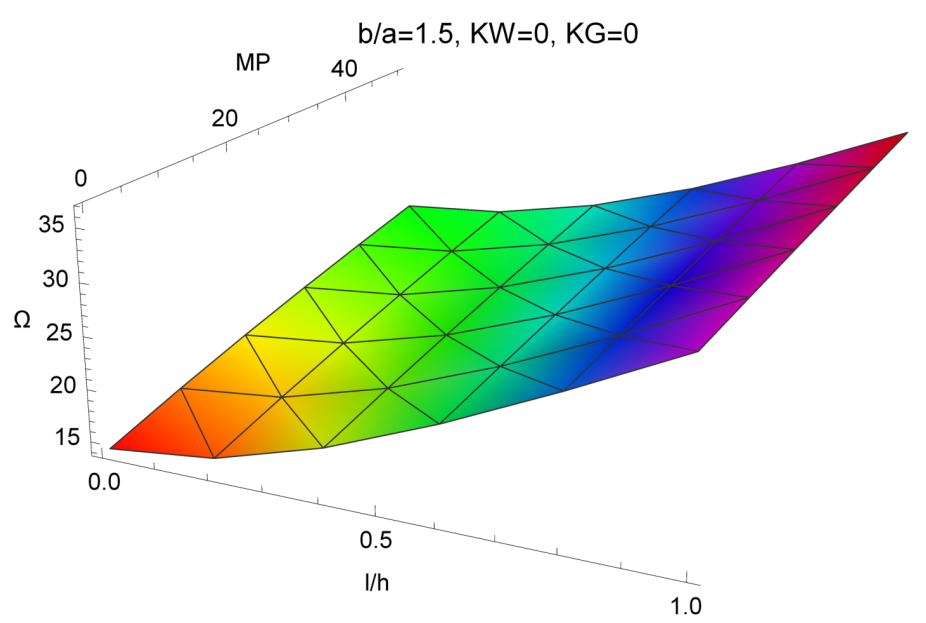

Figure 2. Dimensionless frequencies $\Omega$ in terms of $M P$ and $l / h$ with SSSS boundary conditions.

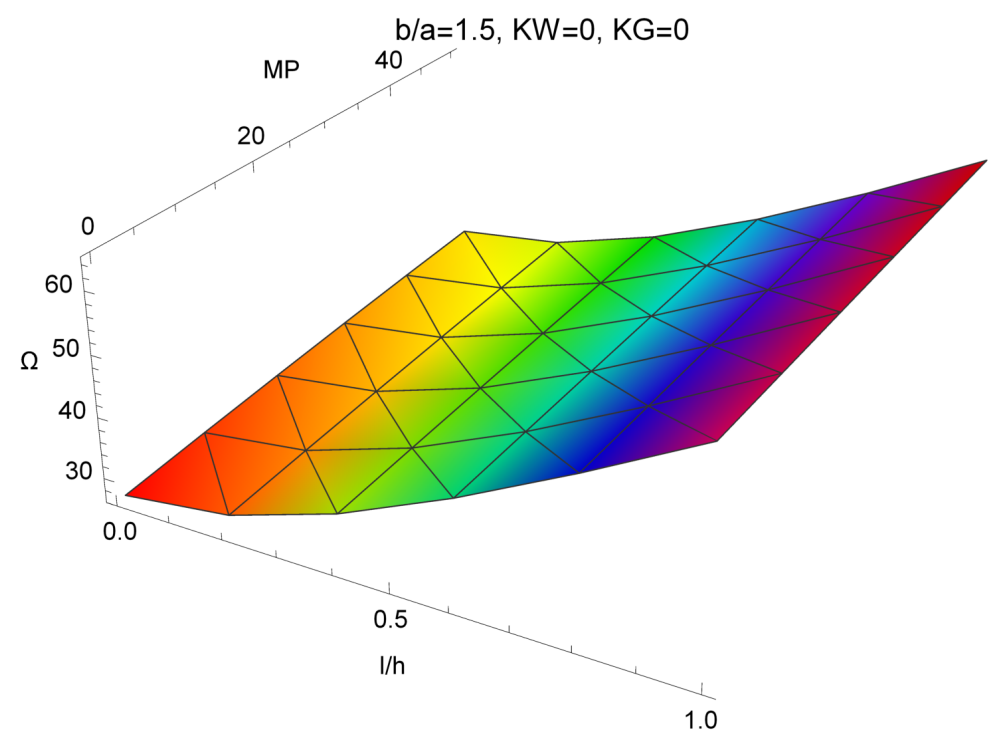

Figure 3. Dimensionless frequencies $\Omega$ in terms of $M P$ and $l / h$ with CCCC boundary conditions.

To study the effect of the elastic foundation using the Winkler-type model, the Winkler modulus parameter $K W$ is taken within the range of $0 . .400$ as in $[23,25]$. The results are presented for the thickness ratio $l / h=0,0.2,0.4,0.6,0.8,1, M P=0, K G=0$ and simply supported boundary conditions (Figure 4). Analysing the results it can be noticed that by an increase of the Winkler modulus, the value of the frequency increases for all values of $l / h$ and the difference in results from the classical theory becomes smaller. Taking the shear modulus factor $K G$ in the range $0-10[23,25]$, we studied influence of the Pasternak-type foundation on the vibration frequency parameter (Figure 5). Calculations are performed by varying $l / h=0,0.2,0.4,0.6,0.8,1$ for fixed $M P=0, K W=100$. Obtained results show an increase of the dimensionless frequency with an increase of the shear modulus. The small scale effect was investigated, changing the type of boundary conditions (Figure 6). Here four types of symmetrical boundary conditions are considered: SSSS, CCCC, CSCS $(x=-a / 2 ; a / 2$ are clamped, $y=-b / 2 ; b / 2$ are simply supported), $\operatorname{SCSC}(x=-a / 2 ; a / 2$ are simply supported, $y=-b / 2 ; b / 2$ are clamped). The graphene sheet is exposed to the magnetic field with the magnetic parameter $M P=10$. We also consider that the Winkler modulus and the shear modulus of the surrounding elastic medium equal to 100 and 10, respectively. Analysis of results allows concluding that an increase in the clamped part of the boundary implies an increase in the frequency value and the greatest values are reached 
under CCCC boundary conditions. The difference in the results with an increase in the thickness ratio $l / h$ is more significant for clamped boundary conditions.

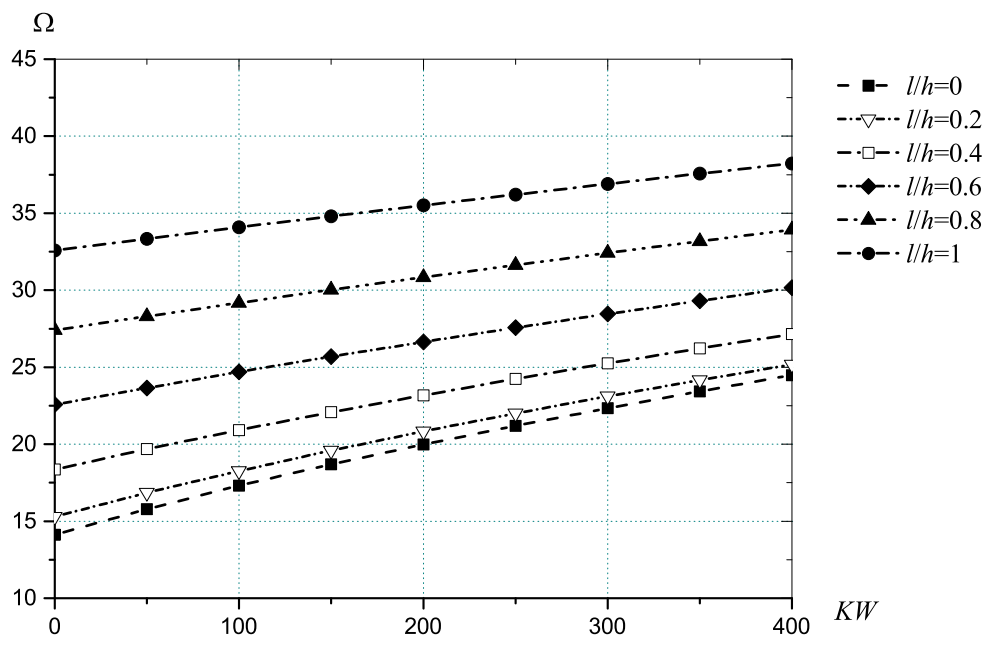

Figure 4. Dimensionless frequencies $\Omega$ depending on Winkler modulus for different thickness ratios $l / h$ and SSSS boundary conditions $(b / a=1.5, K G=0, M P=0)$.

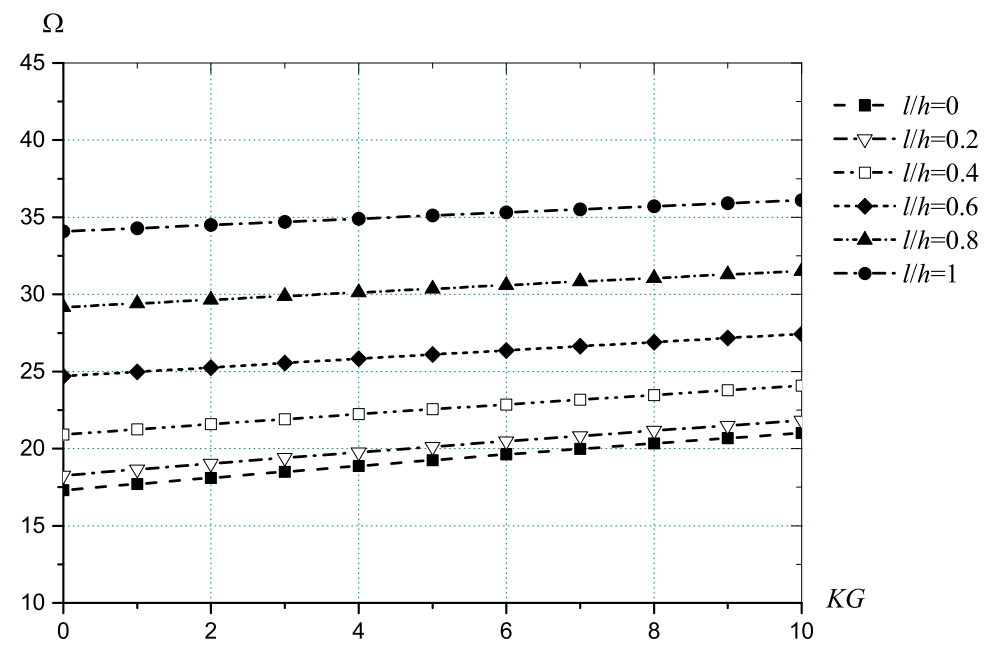

Figure 5. Dimensionless frequencies $\Omega$ depending on shear modulus for different thickness ratios $l / h$ and SSSS boundary conditions $(b / a=1.5, K W=100, M P=0)$. 


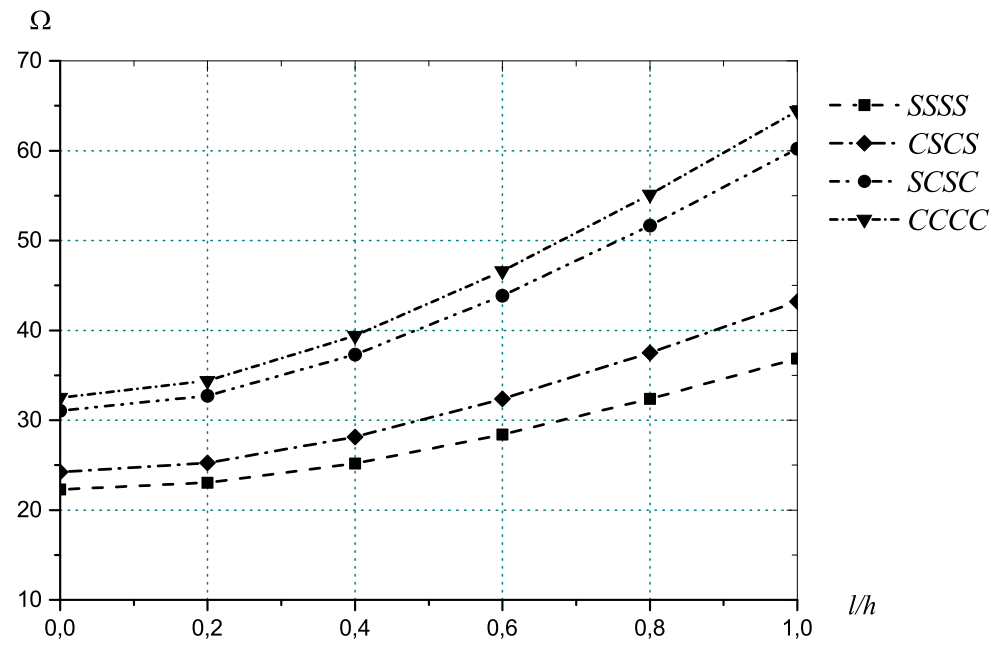

Figure 6. Dimensionless frequencies $\Omega$ for various types of the boundary conditions $(b / a=1.5, K W=$ $100, K G=10, M P=10)$.

\section{Concluding Remarks}

The small-scale analysis of a single-layered rectangular orthotropic plate exposed to a magnetic field is performed. The considered plate is embedded in an elastic medium modeled as the Winkler and the Pasternak foundation. The study is based on the modified couple stress theory and the Kirchhoff-Love hypotheses. The influence of the magnetic field is derived by the Lorentz force. The proposed approach uses the Ritz method. The variational formulation of the considered problem is given, which can be used for future investigation of plates with more complicated shape by changing the shape functions and a region of integration.

The results of the numerical investigation contain the analysis of an influence of the boundary conditions, the magnetic parameter, the parameters of elastic foundation on vibration frequencies. It is concluded that the frequency of a plate is sensitive to the material length scale parameter. The magnetic field plays an important role and significantly increases the vibration frequencies. Simultaneously it is observed that for higher values of the magnetic field strength the effect of the length scale parameter decreases. Thus, the small-scale effect is dampened by an increase in magnetic field strength. An increase in the clamped part of the boundary leads to an increase in the frequency values and a more pronounced small-scale effect. The biggest values of frequencies appear for a plate with all clamped edges.

Author Contributions: Conceptualization, O.M., J.A.; methodology, O.M., J.A.; software, O.M.; validation, O.M., J.A.; formal analysis, O.M., J.A.; investigation, O.M., J.A.; resources, O.M., J.A.; data curation, O.M., J.A.; writing—original draft preparation, O.M., J.A.; writing—review and editing, O.M., J.A.; visualization, O.M., J.A.; supervision, J.A.; project administration, J.A.; funding acquisition, J.A. All authors have read and agreed to the published version of the manuscript.

Funding: This work has been supported by the Polish National Science Centre under the Grant OPUS 14 No. 2017/27/B/ST8/01330.

Conflicts of Interest: The authors declare no conflict of interest. 


\section{Abbreviations}

The following abbreviations are used in this manuscript:

NEMS nano-electro mechanical systems

MEMS micro-electro mechanical systems

SSSS all edges are simply supported

CCCC all edges are clamped

SSSC three edges are simply supported and one is clamped

SCSC two opposite sides are simply supported, the other two ones are clamped

SCSF two opposite sides are simply supported, one is clamped and one is free

SSSF the sides except one are simply supported and one is free

CSCS two opposite sides are clamped, the other two ones are simply supported

\section{References}

1. $\mathrm{Bu}, \mathrm{I} . ;$ Yang, C. High-performance ZnO nanoflake moisture sensor. Superlattices Microstruct. 2012, 51, 745-753. [CrossRef]

2. Hoa, N.; Duy, N. Hieu NCrystalline mesoporous tungsten oxide nanoplate monoliths synthesized by directed soft template method for highly sensitive $\mathrm{NO}_{2}$ gas sensor applications. Mater. Res. Bull. 2013, 48, 440-448. [CrossRef]

3. Kriven, W.; Kwak, S.; Wallig, M.; Choy, J. Bio-resorbable nanoceramics for gene and drug delivery. Mrs Bull. 2004, 29, 33-37. [CrossRef]

4. Bi, L.; Rao, Y.; Tao, Q.; Dong, J.; Su, T.; Liu, F.; Qian, W. Fabrication of large-scale gold nanoplate films as highly active SERS substrates for label-free DNA detection. Biosens. Bioelectron. 2013, 43, 193-199. [CrossRef] [PubMed]

5. Zhong, Y.; Guo, Q.; Li, S.; Shi, J.; Liu, L. Heat transfer enhancement of paraffin wax using graphite foam for thermal energy storage. Sol. Energy Mater. Sol. Cells 2010, 94, 1011-1014. [CrossRef]

6. Lam, D.C.C.; Yang, F.; Chong, A.C.M.; Wang, J.; Tong, P. Experiments and theory in strain gradient elasticity. J. Mech. Phys. Solids 2003, 51, 1477-1508. [CrossRef]

7. Chong, A.C.M.; Yang, F.; Lam, D.C.C.; Tong, P. Torsion and bending of micron-scaled structures. J. Mater. Res. 2001, 16, 1052-1058 [CrossRef]

8. Stölken, J.S.; Evans, A.G. A microbend test method for measuring the plasticity length scale. Acta Mater. 1998, 46, 5109-5115 [CrossRef]

9. Cosserat, E.; Cosserat, F. Theory of Deformable Bodies; Delphenich D.H., Ed.; Scientific Library A. Herman and Sons: Paris, France, 1909.

10. Mindlin, R.D.; Tiersten, H.F. Effects of couple-stresses in linear elasticity. Arch. Ration Mech. Anal. 1962, 11, 415-48. [CrossRef]

11. Toupin, R.A. Elastic materials with couple stresses. Arch. Ration Mech. Anal. 1962, 11, 385-414. [CrossRef]

12. Koiter, W.T. Couple stresses in the theory of elasticity, I and II. Proc. K Ned. Akad. Wet B 1964, 67, 17-44.

13. Eringen, A.C. On differential equations of nonlocal elasticity and solutions of screw dislocation and surface waves. J. Appl. Phys. 1983, 54, 4703-4710. [CrossRef]

14. Yang, F.; Chong, A.C.M.; Lam, D.C.C.; Tong, P. Couple stress based strain gradient theory for elasticity. Int. J. Solids Struct. 2002, 39, 2731-2743. [CrossRef]

15. Tsiatas, G.C. A new Kirchhoff plate model based on a modified couple stress theory. Int. J. Solids Struct. 2009, 46, 2757-64. [CrossRef]

16. Yin, L.; Qian, Q.; Wang, L.; Xia, W. Vibration analysis of microscale plates based on modified couple stress theory. Acta Mech. Solida Sin. 2010, 23, 386-93. [CrossRef]

17. Simsek, M.; Aydın, M.; Yurtcu, H.H.; Reddy, J.N. Size-dependent vibration of a microplate under the action of a moving load based on the modified couple stress theory. Acta Mech. 2015, 226, 3807-3822. [CrossRef]

18. Jomehzadeh, E.; Noori, H.R.; Saidi, A.R. The size-dependent vibration analysis of micro-plates based on a modified couple stress theory. Physica E 2011, 43, 877-883. [CrossRef]

19. Tsiatas, A.J.; Yiotis, G.C. A Microstructure-Dependent Orthotropic Plate Model Based on a Modified Couple Stress Theory Recent Developments in Boundary Element Methods; Sapountzakis, E.J., Ed.; National Technical University of Athens: Athens, Greece, 2010; pp. 295-308. 
20. Ziaee, S. Linear free vibration of micro-/nano-plates with cut-out in thermal environment via modified couple stress theory and Ritz method. Ain Shams Eng. J. 2018, 9, 2373-2381. [CrossRef]

21. Tsiatas, G.C.; Yiotis, A.J. Size effect on the static, dynamic and buckling analysis of orthotropic Kirchhoff-type skew micro-plates based on a modified couple stress theory: comparison with the nonlocal elasticity theory. Acta Mech. 2015, 226, 1267-1281. [CrossRef]

22. Akgöz, B.; Civalek, Ö. Free vibration analysis for single-layered graphene sheets in an elastic matrix via modified couple stress theory. Mater. Des. 2012, 42, 164-171. [CrossRef]

23. Bastami, M.; Behjat, B. Ritz Solution of Buckling and Vibration Problem of nanoplates embedded in an elastic medium. Sigma J. Eng. Nat. Sci. 2017, 35, 285-302.

24. Mohammadi, M.; Moradi, A.; Ghayour, M.; Farajpour, A. Exact solution for thermo-mechanical vibration of orthotropic mono-layer graphene sheet embedded in an elastic medium. Lat. Am. J. Solids Struct. 2014, 11, 437-458. [CrossRef]

25. Mohammadi, M.; Goodarzi, M.; Ghayour; M; Alivand, S. Small Scale effect on the vibration of orthotropic plates embedded in an elastic medium and under biaxial in-plane pre-load via nonlocal elasticity theory. J. Solid Mech. 2012, 4, 128-143.

26. Behfar, K.; Naghdabadi, R. Nanoscale vibrational analysis of a multi-layered graphene sheet embedded in an elastic medium. Compos. Sci. Technol. 2005, 65, 1159-1164. [CrossRef]

27. Choi, E.; Brooks, J.; Eaton DAl-Haik, M.; Hussaini, M.; Garmestani, H.; Li DDahmen, K. Enhancement of thermal and electrical properties of carbon nanotube polymer composites by magnetic field processing. J. Appl. Phys. 2003, 94, 6034-6039. [CrossRef]

28. Lee, S.; Ma, C.; Yuen, S.; Teng, C.; Liao, S.; Huang, Y.; Tsai, M.; Su, A.; Wang, I. Magnetic field enhances the graphitized structure and field emission effect of carbon nanotubes. Thin Solid Film. 2011, 519, 4166-4173. [CrossRef]

29. Faugeras, C.; Kossacki, P.; Basko, D.; Amado, M.; Sprinkle, M.; Berger, C.; De Heer, W.; Potemski, M. Effect of a magnetic field on the two-phonon Raman scattering in graphene. Phys. Rev. B Condens. Matter Mater. Phys. 2010, 81, 155436 [CrossRef]

30. Wang, Y.; Hoang, Y.; Song, Y.; Zhang, X.; Ma, Y.; Liang, J.; Chen, Y. Room-temperature ferromagnetism of graphene. Nano Lett. 2009, 9, 220-224. [CrossRef]

31. Murmu, T.; McCarthy, M.A.; Adhikari, S. In-plane magnetic field affected transverse vibration of embedded single-layer graphene sheets using equivalent nonlocal elasticity approach. Compos. Struct. 2013, 96, 57-63. [CrossRef]

32. Kiani, K. Revisiting the free transverse vibration of embedded single-layer graphene sheets acted upon by an in-plane magnetic field. J. Mech. Sci. Technol. 2014, 28, 3511-3516. [CrossRef]

33. Ghorbanpour Arani, A.H.; Maboudi, M.J.; Ghorbanpour Arani, A.; Amir, S. 2D-Magnetic Field and Biaxiall In-Plane Pre-Load Effects on the Vibration of Double Bonded Orthotropic Graphene Sheets. J. Solid Mech. 2013, 5, 193-205.

34. Atanasov, M.S.; Karlicic, D.; Kozic, P. Forced transverse vibrations of an elastically connected nonlocal orthotropic double-nanoplate system subjected to an in-plane magnetic field. Acta Mech. 2017, 228, 2165-2185. [CrossRef]

35. Vol'mir, A.S. Nonlinear Dynamics of Plates and Shells; Nauka: Moscow, Russia, 1972.

36. Baferani, A.H.; Saidi, A.R.; Ehteshami, H. Accurate solution for free vibration analysis of functionally graded thick rectangular plates resting on elastic foundation. Compos. Struct. 2011, 93, 1842-1853. [CrossRef]

37. Parida, S.; Mohanty, S.C. Nonlinear free vibration analysis of functionally graded plate resting on elastic foundation in thermal environment using higher-order shear deformation theory. Sci. Iran. B 2019, 26, 815-833. [CrossRef]

38. Rossi, R.E.; Bambill, D.V.; Laura, P.A.A. Vibrations of a rectangular orthotropic plate with a free edge: A comparison of analytical and numerical results. Ocean Eng. 1998, 25, 521-527. [CrossRef]

(C) 2020 by the authors. Licensee MDPI, Basel, Switzerland. This article is an open access article distributed under the terms and conditions of the Creative Commons Attribution (CC BY) license (http:/ / creativecommons.org/licenses/by/4.0/). 